International Journal of Pure and Applied Mathematics

Volume 100 No. 2 2015, 211-224

ISSN: 1311-8080 (printed version); ISSN: 1314-3395 (on-line version)

url: http://www.ijpam.eu

doi: http://dx.doi.org/10.12732/ijpam.v100i2.4

ijpam.eu

\title{
ESTIMATION OF VARIANCE OF TIME TO RECRUITMENT \\ FOR A TWO GRADE MANPOWER SYSTEM WITH TWO \\ SOURCES OF DEPLETION AND TWO TYPES OF POLICY \\ DECISIONS WHEN THE BREAKDOWN THRESHOLD \\ HAS THREE COMPONENTS
}

\author{
S. Dhivya ${ }^{1 \S}$, A. Srinivasan ${ }^{2}$ \\ ${ }^{1}$ Department of Mathematics \\ Srinivasan Engineering College \\ Perambalur, 621 212, Tamil Nadu, INDIA \\ ${ }^{2} \mathrm{PG} \&$ Research department of Mathematics \\ Bishop Heber College \\ Trichy, 17, Tamil Nadu, INDIA
}

\begin{abstract}
A marketing organization consisting of two grades with policy and transfer decisions forming two different sources of depletion is considered. The policy decisions are classified into two different types according to the intensity of attrition. In this paper the problem of time to recruitment for this organization is analysed when the breakdown threshold, a level of maximum allowable manpower depletion, has three components namely normal threshold of depletion of manpower, threshold of frequent breaks of existing workers and threshold of backup or reservation of manpower sources. A mathematical model is constructed and using a univariate policy of recruitment based on shock model approach, analytical results are obtained to estimate the variance of the time to recruitment by considering different forms of the breakdown threshold for the cumulative loss of manpower in the organization. The results are numerically illustrated by assuming specific distributions and relevant findings are presented.
\end{abstract}

AMS Subject Classification: 990B70, 91B40, 91D35

Received: August 17, 2014

(c) 2015 Academic Publications, Ltd.

$\S$ Correspondence author 
Key Words: two grade manpower system, two sources of depletion of manpower, two types of policy decisions, breakdown threshold of depletion with three components, shock model approach, univariate policy of recruitment and performance measures

\section{Introduction}

In any organization depletion of manpower is quite common when a policy decision is announced. Two sources of depletion of manpower are considered here. Decisions on pay, perquisites and work target form the first source and transfer of personal to sister organizations form the second source of depletion. As immediate recruitment after depletion of each personal is not advisable due to cost and time consumption, recruitment is postponed to a point of time beyond which normal activities cannot be continued due to shortage of manpower. This level of allowable manpower depletion is called breakdown threshold. Elangovan et.al [6] have initiated the study on recruitment problem for a single grade manpower system with two sources of depletion and obtained the variance of time to recruitment using univariate CUM policy of recruitment when the loss of man power in the organization due to the two sources of depletion, interpolicy decision times, inter-transfer decision times and threshold for the loss of man power in the organization, each forming a sequence of independent and identically exponential random variables with different means. Sathiya and Srinivasan [8] have studied the work of Elangovan when the loss of manpower follow poisson (Geometric) distribution. Later Suganya and Srinivasan [9] have extended the work of sathiya and Srinivasan for two grade manpower system. Recently, Dhivya and Srinivasan [2],[3],[4] and [5] have extended the work of Elangovan et.al for a two grade manpower system under different conditions on the inter-policy decisions, inter-transfer decisions and thresholds for the cumulative loss of manpower in each grade. Arivazhagan et.al [1] have determined the mean time to recruitment for a single manpower system with policy decisions forming the only one source of depletion when the threshold for the cumulative loss of manpower has three components. In all the above cited works, it is assumed that all policy decisions have same attrition rate. This assumption on policy decisions is not realistic since policy decisions can have high or low intensity of attrition. This aspect of policy decisions having high or low intensity of attrition is taken into account here. The objective of the present paper is to extend the work of Arivazhagan et.al [1] for a two grade manpower system with two sources of depletion when the policy decisions are classified into two 
types according to the intensity of attrition.

\section{Model Description}

Consider an organization with two grades taking policy and transfer decisions at random epochs in $(0, \infty)$. The policy decisions are classified into two types depending upon their intensity of attrition. Let $p$ be proportion of policy decisions having high attrition rate $\mu_{h}$ and $(1-p)$ be the proportion of policy decisions having low attrition rate $\mu_{l}$. The most suitable distribution of interpolicy decision times is hyper exponential with distribution $F($.$) , probability$ density function $f($.$) . Let the inter-transfer decision times be independent and$ identically distributed exponential random variables with distribution $W($.$) ,$ probability density function $w($.$) and mean \frac{1}{\mu_{2}}\left(\mu_{2}>0\right)$. It is assumed that the two sources of depletion are independent. Let $f_{m}($.$) be the m-fold convolution$ of $f($.$) with itself and w_{n}($.$) be the n-fold convolution of w($.$) with itself. At$ every policy decision making epoch a random number of persons quit the organizations and at every transfer decision making epoch a random number of persons are transferred to the sister organizations. There is an associated loss of manpower in each grade, if a person quits or transferred. It is assumed that the loss of manpower is linear and cumulative. For $i=1,2,3 \ldots$,let $X_{A i}$ and $X_{B i}$ be the continuous random variables representing the amount of depletion of manpower(loss of man hours) in grades $A$ and $B$ respectively caused due to the $i^{\text {th }}$ policy decision. It is assumed that $X_{A i}$ and $X_{B i}$ are independent for each i and each form a sequence of independent and identically distributed random variables with distributions $G_{A}($.$) and G_{B}($.$) and probability density$ functions $g_{A}($.$) and g_{B}($.$) respectively. Let \bar{X}_{A m}$ and $\bar{X}_{B m}$ be the cumulative depletion of manpower in the first $\mathrm{m}$ policy decisions in grades $A$ and $B$ respectively. Let $\bar{X}_{m}$ be the cumulative depletion of manpower in the organization due to the first $m$ policy decisions. For $j=1,2 \ldots$, let $Y_{A j}$ and $Y_{B j}$ be the continuous random variables representing the amount of depletion of manpower in grades $A$ and $B$ respectively caused due to the $j^{\text {th }}$ transfer decision. It is assumed that $Y_{A j}$ and $Y_{B j}$ are independent for each $j$ and each form a sequence of independent and identically distributed random variables with probability density functions $h_{A}($.$) and h_{B}($.$) respectively. Let \bar{Y}_{A n}$ and $\bar{Y}_{B n}$ be the cumulative depletion of manpower in the first $n$ transfer decisions in grades $A$ and $B$ respectively. Let $\bar{Y}_{n}$ be the cumulative depletion of manpower in the organization due to the first $n$ transfer decisions. For each $i$ and $j X_{A i}, X_{B i}, Y_{A j}$ and $Y_{B j}$ are statistically independent. Let $\bar{g}_{A}($.$) and \bar{g}_{B}($.$) be$ 
the Laplace transforms of $g_{A}($.$) and g_{B}($.$) respectively. Let Z$ be the breakdown threshold level for the cumulative loss of manpower in the organization. For grade $A$, let $Z_{A 1}$ be the normal exponential threshold of depletion of manpower with mean $\frac{1}{\theta_{A 1}}\left(\theta_{A 1}>0\right), Z_{A 2}$ be the exponential threshold of frequent breaks of existing workers with mean $\frac{1}{\theta_{A 2}}\left(\theta_{A 2}>0\right)$ and $Z_{A 3}$ be the threshold of backup or reservation of manpower sources with mean $\frac{1}{\theta_{A 3}}\left(\theta_{A 3}>0\right)$. For grade $B$, let $Z_{B 1}, Z_{B 2}$ and $Z_{B 3}$ be the normal exponential threshold of depletion of manpower, exponential threshold of frequent breaks of existing workers and exponential threshold of backup or reservation of manpower sources with means $\frac{1}{\theta_{B 1}}, \frac{1}{\theta_{B 2}}$ and $\frac{1}{\theta_{B 3}}\left(\theta_{B 1}, \theta_{B 2}\right.$ and $\left.\theta_{B 3}>0\right)$ respectively. Let $k($.$) be the probability$ density function of $Z$. The univariate CUM policy of recruitment employed in this paper is stated as follows:

Recruitment is done whenever the cumulative loss of man hours in the organization exceeds the breakdown threshold.

Let $T$ be the random variable denoting the time to recruitment with distribution $L($.$) , probability density function l($.$) , Laplace transform \bar{l}(s)$, mean $E(T)$ and variance $V(T)$. Let $N_{P}(T)$ be the number of policy decisions required to make recruitment at $T$ and $N_{\text {Trans. }}(T)$ be the number of transfer decisions required to make recruitment at $T$. Let $\bar{X}_{N_{P}(T)}$ and $\bar{Y}_{N_{\text {Trans. }}(T)}$ be the respective total loss of manpower in $N_{P}(T)$ and $N_{\text {Trans. }}(T)$ decisions incurred by the organization during $(0, T)$.

\section{Main Results}

The survivor function of $T$ is

$$
P(T>t)=P\left(\bar{X}_{N_{P}(T)}+\bar{Y}_{N_{\text {Trans. }}(T)}<Z\right)
$$

Since $\left\{N_{P}(T)\right\}$ and $\left\{N_{\text {Trans. }}(T)\right\}$ form two independent ordinary renewal processes, from Renewal theory [7] and (1) we find that

$$
P(T>t)=\sum_{m=0}^{\infty} \sum_{n=0}^{\infty}\left[F_{m}(t)-F_{m+1}(t)\right]\left[W_{n}(t)-W_{n+1}(t)\right] P\left(\bar{X}_{m}+\bar{Y}_{n} \leq Z\right)
$$

where

$$
P\left(\bar{X}_{m}+\bar{Y}_{n} \leq Z\right)=\int_{0}^{\infty} P\left(\bar{X}_{m}+\bar{Y}_{n}<z\right) k(z) \mathrm{d} z .
$$

We now estimate the mean and variance of $T$ for different cases: 
Case (i).

$$
Z=\min \left(Z_{A 1}+Z_{A 2}+Z_{A 3}, Z_{B 1}+Z_{B 2}+Z_{B 3}\right)
$$

Since

$$
P(Z>z)=\left\{P\left(Z_{A 1}+Z_{A 2}+Z_{A 3}>z\right) P\left(Z_{B 1}+Z_{B 2}+Z_{B 3}>z\right)\right\},
$$

from the hypothesis and on simplification it can be shown that

$$
\begin{aligned}
k(z)= & C_{1}\left(\theta_{A 1}+\theta_{B 1}\right) e^{-\left(\theta_{A 1}+\theta_{B 1}\right) z}+C_{2}\left(\theta_{A 2}+\theta_{B 2}\right) e^{-\left(\theta_{A 2}+\theta_{B 2}\right) z}+ \\
& C_{3}\left(\theta_{A 3}+\theta_{B 3}\right) e^{-\left(\theta_{A 3}+\theta_{B 3}\right) z}-C_{4}\left(\theta_{A 1}+\theta_{B 2}\right) e^{-\left(\theta_{A 1}+\theta_{B 2}\right) z}+ \\
& C_{5}\left(\theta_{A 1}+\theta_{B 3}\right) e^{-\left(\theta_{A 1}+\theta_{B 3}\right) z}-C_{6}\left(\theta_{A 2}+\theta_{B 1}\right) e^{-\left(\theta_{A 2}+\theta_{B 1}\right) z}- \\
& C_{7}\left(\theta_{A 2}+\theta_{B 3}\right) e^{-\left(\theta_{A 2}+\theta_{B 3}\right) z}+C_{8}\left(\theta_{A 3}+\theta_{B 1}\right) e^{-\left(\theta_{A 3}+\theta_{B 1}\right) z}- \\
& C_{9}\left(\theta_{A 3}+\theta_{B 2}\right) e^{-\left(\theta_{A 3}+\theta_{B 2}\right) z}
\end{aligned}
$$

where

$$
\begin{aligned}
& C_{1}=\frac{\theta_{A 2} \theta_{A 3} \theta_{B 2} \theta_{B 3}}{\left(\theta_{A 2}-\theta_{A 1}\right)\left(\theta_{A 3}-\theta_{A 1}\right)\left(\theta_{B 2}-\theta_{B 1}\right)\left(\theta_{B 3}-\theta_{B 1}\right)}, \\
& C_{2}=\frac{\theta_{A 1} \theta_{A 3} \theta_{B 1} \theta_{B 3}}{\left(\theta_{A 2}-\theta_{A 1}\right)\left(\theta_{A 3}-\theta_{A 2}\right)\left(\theta_{B 2}-\theta_{B 1}\right)\left(\theta_{B 3}-\theta_{B 2}\right)}, \\
& C_{3}=\frac{\theta_{A 1} \theta_{A 2} \theta_{B 1} \theta_{B 2}}{\left(\theta_{A 3}-\theta_{A 2}\right)\left(\theta_{A 3}-\theta_{A 1}\right)\left(\theta_{B 3}-\theta_{B 2}\right)\left(\theta_{B 3}-\theta_{B 1}\right)}, \\
& C_{4}=\frac{\theta_{A 2} \theta_{A 3} \theta_{B 1} \theta_{B 3}}{\left(\theta_{A 2}-\theta_{A 1}\right)\left(\theta_{A 3}-\theta_{A 1}\right)\left(\theta_{B 2}-\theta_{B 1}\right)\left(\theta_{B 3}-\theta_{B 2}\right)}, \\
& C_{5}=\frac{\theta_{A 2} \theta_{A 3} \theta_{B 1} \theta_{B 2}}{\left(\theta_{A 2}-\theta_{A 1}\right)\left(\theta_{A 3}-\theta_{A 1}\right)\left(\theta_{B 3}-\theta_{B 2}\right)\left(\theta_{B 3}-\theta_{B 1}\right)}, \\
& C_{6}=\frac{\theta_{A 1} \theta_{A 3} \theta_{B 2} \theta_{B 3}}{\left(\theta_{A 2}-\theta_{A 1}\right)\left(\theta_{A 3}-\theta_{A 2}\right)\left(\theta_{B 2}-\theta_{B 1}\right)\left(\theta_{B 3}-\theta_{B 1}\right)}, \\
& C_{7}=\frac{\theta_{A 1} \theta_{A 3} \theta_{B 1} \theta_{B 2}}{\left(\theta_{A 2}-\theta_{A 1}\right)\left(\theta_{A 3}-\theta_{A 2}\right)\left(\theta_{B 3}-\theta_{B 2}\right)\left(\theta_{B 3}-\theta_{B 1}\right)}, \\
& C_{8}=\frac{\theta_{A 1} \theta_{A 2} \theta_{B 2} \theta_{B 3}}{\left(\theta_{A 3}-\theta_{A 2}\right)\left(\theta_{A 3}-\theta_{A 1}\right)\left(\theta_{B 2}-\theta_{B 1}\right)\left(\theta_{B 3}-\theta_{B 1}\right)}, \\
& C_{9}=\frac{\theta_{A 1} \theta_{A 2} \theta_{B 1} \theta_{B 3}}{\left(\theta_{A 3}-\theta_{A 2}\right)\left(\theta_{A 3}-\theta_{A 1}\right)\left(\theta_{B 2}-\theta_{B 1}\right)\left(\theta_{B 3}-\theta_{B 2}\right)}
\end{aligned}
$$

From (2), (3) and (4) we get

$$
\begin{aligned}
P(T>t)= & C_{1} D_{\theta_{A 1}, \theta_{B 1}}(t)+C_{2} D_{\theta_{A 2}, \theta_{B 2}}(t)+C_{3} D_{\theta_{A 3}, \theta_{B 3}}(t) \\
& -C_{4} D_{\theta_{A 1}, \theta_{B 2}}(t)+C_{5} D_{\theta_{A 1}, \theta_{B 3}}(t)-C_{6} D_{\theta_{A 2}, \theta_{B 1}}(t) \\
& -C_{7} D_{\theta_{A 2}, \theta_{B 3}}(t)+C_{8} D_{\theta_{A 3}, \theta_{B 1}}(t)-C_{9} D_{\theta_{A 3}, \theta_{B 2}}(t)
\end{aligned}
$$




$$
\text { where } \begin{aligned}
D_{\alpha, \beta}(t)= & \left(\sum_{m=0}^{\infty}\left[F_{m}(t)-F_{m+1}(t)\right]\left[\bar{g}_{A}(\alpha+\beta) \bar{g}_{B}(\alpha+\beta)\right]^{m}\right) \\
& \left(\sum_{n=0}^{\infty}\left[W_{m}(t)-W_{m+1}(t)\right]\left[\bar{h}_{A}(\alpha+\beta) \bar{h}_{B}(\alpha+\beta)\right]^{n}\right)
\end{aligned}
$$

Therefore from (6)

$$
\begin{aligned}
& L(t)=1-P(T>t)=1-\sum_{i=1}^{9} p_{i}(t) \\
& l(t)=-\sum_{i=1}^{9} p_{i}^{\prime}(t) \text { and } \bar{l}(s)=-\sum_{i=1}^{9} \overrightarrow{p_{i}}(s)
\end{aligned}
$$

where $p_{i}(t)$ is the $i^{t h}$ term in the right side of (6) and $p_{i}^{\prime}(t)=\frac{d}{d t} p_{i}(t), i=$ $1,2, \ldots .9$.

The mean and variance of time to recruitment can be computed from (7) and from the result

$$
\begin{aligned}
E(T)=-\left[\frac{d}{d s}[\bar{l}(s)]\right]_{s=0}=\sum_{i=1}^{9}\left[\frac{d}{d s}\left[\overrightarrow{p_{i}}(s)\right]\right]_{s=0}, \quad E\left(T^{2}\right) & =\left[\frac{d^{2}}{d s^{2}}[\bar{l}(s)]\right]_{s=0} \\
& =\sum_{i=1}^{9}\left[\frac{d^{2}}{d s^{2}}\left[\overrightarrow{p_{i}}(s)\right]\right]_{s=0}
\end{aligned}
$$

We now compute $\left[\frac{d}{d s}\left[\overrightarrow{p_{i}}(s)\right]\right]_{s=0}$ and $\left[\frac{d^{2}}{d s^{2}}\left[\overrightarrow{p_{i}}(s)\right]\right]_{s=0}$ for $i=1$. The computation for $\mathrm{i}=2,3, \ldots, 9$ is similar.

On simplification, the first term of right side of (6) is found to be

$$
\begin{aligned}
P_{1}(t)= & C_{1} D_{\theta_{A 1}, \theta_{B 1}}(t) \\
= & C_{1}\left\{1-\left[1-\bar{g}_{A}\left(\theta_{A 1}+\theta_{B 1}\right) \bar{g}_{B}\left(\theta_{A 1}+\theta_{B 1}\right)\right] \sum_{m=1}^{\infty} F_{m}(t)\left[\bar{g}_{A}\left(\theta_{A 1}+\theta_{B 1}\right) \bar{g}_{B}\left(\theta_{A 1}+\theta_{B 1}\right)\right]^{m-1}+\right. \\
& {\left[1-\bar{h}_{A}\left(\theta_{A 1}+\theta_{B 1}\right) \bar{h}_{B}\left(\theta_{A 1}+\theta_{B 1}\right)\right] \sum_{n=1}^{\infty} W_{n}(t)\left[\bar{h}_{A}\left(\theta_{A 1}+\theta_{B 1}\right) \bar{h}_{B}\left(\theta_{A 1}+\theta_{B 1}\right)\right]^{n-1} } \\
& -\left[1-\bar{g}_{A}\left(\theta_{A 1}+\theta_{B 1}\right) \bar{g}_{B}\left(\theta_{A 1}+\theta_{B 1}\right)\right]\left[1-\bar{h}_{A}\left(\theta_{A 1}+\theta_{B 1}\right) \bar{h}_{B}\left(\theta_{A 1}+\theta_{B 1}\right)\right] \\
& \left.\sum_{m=1}^{\infty} F_{m}(t)\left[\bar{g}_{A}\left(\theta_{A 1}+\theta_{B 1}\right) \bar{g}_{B}\left(\theta_{A 1}+\theta_{B 1}\right)\right]^{m-1} \sum_{n=1}^{\infty} W_{n}(t)\left[\bar{h}_{A}\left(\theta_{A 1}+\theta_{B 1}\right) \bar{h}_{B}\left(\theta_{A 1}+\theta_{B 1}\right)\right]^{n-1}\right\}
\end{aligned}
$$


Since $w_{n}(t)=\frac{\mu_{2}^{n} e^{-\mu_{2} t} t^{n-1}}{(n-1) !}$ by hypothesis, we find that

$$
\begin{array}{r}
{\left[1-\bar{h}_{A}\left(\theta_{A 1}+\theta_{B 1}\right) \bar{h}_{B}\left(\theta_{A 1}+\theta_{B 1}\right)\right] \sum_{n=1}^{\infty} W_{n}(t)\left[\bar{h}_{A}\left(\theta_{A 1}+\theta_{B 1}\right) \bar{h}_{B}\left(\theta_{A 1}+\theta_{B 1}\right)\right]^{n-1}} \\
=\mu_{2}\left[1-\bar{h}_{A}\left(\theta_{A 1}+\theta_{B 1}\right) \bar{h}_{B}\left(\theta_{A 1}+\theta_{B 1}\right)\right] e^{-\mu_{2}\left[1-\bar{h}_{A}\left(\theta_{A 1}+\theta_{B 1}\right) \bar{h}_{B}\left(\theta_{A 1}+\theta_{B 1}\right)\right] t}
\end{array}
$$

From (9) and (10) and on simplification we get

$$
\begin{aligned}
& {\left[\frac{d}{d s}\left[\overline{p_{1}^{\prime}}(s)\right]\right]_{s=0}=\frac{C_{1}\left[1-\bar{f}\left(\mu_{2}\left(1-\bar{h}_{A}\left(\theta_{A 1}+\theta_{B 1}\right) \bar{h}_{B}\left(\theta_{A 1}+\theta_{B 1}\right)\right)\right)\right]}{\mu_{2}\left(1-\bar{h}_{A}\left(\theta_{A 1}+\theta_{B 1}\right) \bar{h}_{B}\left(\theta_{A 1}+\theta_{B 1}\right)\right)\left[1-\bar{f}\left(\mu_{2}\left(1-\bar{h}_{A}\left(\theta_{A 1}+\theta_{B 1}\right) \bar{h}_{B}\left(\theta_{A 1}+\theta_{B 1}\right)\right)\right)\right.}} \\
& -C_{1}\left[2 \mu_{2}\left(1-\bar{h}_{A}\left(\theta_{A 1}+\theta_{B 1}\right) \bar{h}_{B}\left(\theta_{A 1}+\theta_{B 1}\right)\right)\right. \\
& \left(1-\bar{g}_{A}\left(\theta_{A 1}+\theta_{B 1}\right) \bar{g}_{B}\left(\theta_{A 1}+\theta_{B 1}\right)\right) \\
& \bar{f}\left(\mu_{2}\left(1-\bar{h}_{A}\left(\theta_{A 1}+\theta_{B 1}\right) \bar{h}_{B}\left(\theta_{A 1}+\theta_{B 1}\right)\right)\right)+ \\
& 2\left[1-\bar{f}\left(\mu_{2}\left(1-\bar{h}_{A}\left(\theta_{A 1}+\theta_{B 1}\right) \bar{h}_{B}\left(\theta_{A 1}+\theta_{B 1}\right)\right)\right)\right. \\
& \left.\bar{g}_{A}\left(\theta_{A 1}+\theta_{B 1}\right) \bar{g}_{B}\left(\theta_{A 1}+\theta_{B 1}\right)\right] \\
& {\left[\frac{d^{2}}{d s^{2}}\left[\overline{p_{1}}(s)\right]\right]_{s=0}=\frac{\left.\left[1-\bar{f}\left(\mu_{2}\left(1-\bar{h}_{A}\left(\theta_{A 1}+\theta_{B 1}\right) \bar{h}_{B}\left(\theta_{A 1}+\theta_{B 1}\right)\right)\right)\right]\right]}{\mu_{2}^{2}\left(1-\bar{h}_{A}\left(\theta_{A 1}+\theta_{B 1}\right) \bar{h}_{B}\left(\theta_{A 1}+\theta_{B 1}\right)\right)^{2}}} \\
& {\left[1-\bar{f}\left(\mu_{2}\left(1-\bar{h}_{A}\left(\theta_{A 1}+\theta_{B 1}\right) \bar{h}_{B}\left(\theta_{A 1}+\theta_{B 1}\right)\right)\right)\right.} \\
& \left.\bar{g}_{A}\left(\theta_{A 1}+\theta_{B 1}\right) \bar{g}_{B}\left(\theta_{A 1}+\theta_{B 1}\right)\right]^{2}
\end{aligned}
$$

where

$$
\begin{aligned}
\bar{f}\left(\mu _ { 2 } \left(1-\bar{h}_{A}\left(\theta_{A 1}\right.\right.\right. & \left.\left.\left.+\theta_{B 1}\right) \bar{h}_{B}\left(\theta_{A 1}+\theta_{B 1}\right)\right)\right) \\
= & \frac{p \mu_{h}}{\mu_{2}\left(1-\bar{h}_{A}\left(\theta_{A 1}+\theta_{B 1}\right) \bar{h}_{B}\left(\theta_{A 1}+\theta_{B 1}\right)\right)+\mu_{h}} \\
& \quad+\frac{(1-p) \mu_{l}}{\mu_{2}\left(1-\bar{h}_{A}\left(\theta_{A 1}+\theta_{B 1}\right) \bar{h}_{B}\left(\theta_{A 1}+\theta_{B 1}\right)\right)+\mu_{l}}
\end{aligned}
$$

and

$$
\begin{aligned}
\bar{f}^{\prime}\left(\mu_{2}\left(1-\bar{h}_{A}\left(\theta_{A 1}+\theta_{B 1}\right) \bar{h}_{B}\left(\theta_{A 1}+\theta_{B 1}\right)\right)\right) & \\
= & \frac{-p \mu_{h}}{\left[\mu_{2}\left(1-\bar{h}_{A}\left(\theta_{A 1}+\theta_{B 1}\right) \bar{h}_{B}\left(\theta_{A 1}+\theta_{B 1}\right)\right)+\mu_{h}\right]^{2}} \\
& -\frac{(1-p) \mu_{l}}{\left[\mu_{2}\left(1-\bar{h}_{A}\left(\theta_{A 1}+\theta_{B 1}\right) \bar{h}_{B}\left(\theta_{A 1}+\theta_{B 1}\right)\right)+\mu_{l}\right]^{2}}
\end{aligned}
$$


by hypothesis.

From (8),(11) and (12) we get

$$
\begin{aligned}
E(T)= & C_{1} E_{\theta_{A 1}, \theta_{B 1}}+C_{2} E_{\theta_{A 2}, \theta_{B 2}}+C_{3} E_{\theta_{A 3}, \theta_{B 3}} \\
& -C_{4} E_{\theta_{A 1}, \theta_{B 2}}+C_{5} E_{\theta_{A 1}, \theta_{B 3}}-C_{6} E_{\theta_{A 2}, \theta_{B 1}} \\
& -C_{7} E_{\theta_{A 2}, \theta_{B 3}}+C_{8} E_{\theta_{A 3}, \theta_{B 1}}-C_{9} E_{\theta_{A 3}, \theta_{B 2}}
\end{aligned}
$$

and

$$
\begin{aligned}
E\left(T^{2}\right)= & C_{1} E_{\theta_{A 1}, \theta_{B 1}}^{2}+C_{2} E_{\theta_{A 2}, \theta_{B 2}}^{2}+C_{3} E_{\theta_{A 3}, \theta_{B 3}}^{2} \\
& -C_{4} E_{\theta_{A 1}, \theta_{B 2}}^{2}+C_{5} E_{\theta_{A 1}, \theta_{B 3}}^{2}-C_{6} E_{\theta_{A 2}, \theta_{B 1}}^{2} \\
& -C_{7} E_{\theta_{A 2}, \theta_{B 3}}^{2}+C_{8} E_{\theta_{A 3}, \theta_{B 1}}^{2}-C_{9} E_{\theta_{A 3}, \theta_{B 2}}^{2}
\end{aligned}
$$

(13) and (14) give the variance of the time to recruitment for this case.

Case (ii)

$$
Z=\max \left(Z_{A 1}+Z_{A 2}+Z_{A 3}, Z_{B 1}+Z_{B 2}+Z_{B 3}\right)
$$

Since

$$
P(Z \leq z)=\left\{P\left(Z_{A 1}+Z_{A 2}+Z_{A 3} \leq z\right) P\left(Z_{B 1}+Z_{B 2}+Z_{B 3} \leq z\right)\right\}
$$

from the hypothesis and on simplification it can be shown that

$$
\begin{aligned}
k(z)= & -C_{1}\left(\theta_{A 1}+\theta_{B 1}\right) e^{-\left(\theta_{A 1}+\theta_{B 1}\right) z}-C_{2}\left(\theta_{A 2}+\theta_{B 2}\right) e^{-\left(\theta_{A 2}+\theta_{B 2}\right) z}- \\
& C_{3}\left(\theta_{A 3}+\theta_{B 3}\right) e^{-\left(\theta_{A 3}+\theta_{B 3}\right) z}+C_{4}\left(\theta_{A 1}+\theta_{B 2}\right) e^{-\left(\theta_{A 1}+\theta_{B 2}\right) z}- \\
& C_{5}\left(\theta_{A 1}+\theta_{B 3}\right) e^{-\left(\theta_{A 1}+\theta_{B 3}\right) z}+C_{6}\left(\theta_{A 2}+\theta_{B 1}\right) e^{-\left(\theta_{A 2}+\theta_{B 1}\right) z}+ \\
& C_{7}\left(\theta_{A 2}+\theta_{B 3}\right) e^{-\left(\theta_{A 2}+\theta_{B 3}\right) z}-C_{8}\left(\theta_{A 3}+\theta_{B 1}\right) e^{-\left(\theta_{A 3}+\theta_{B 1}\right) z}+ \\
& C_{9}\left(\theta_{A 3}+\theta_{B 2}\right) e^{-\left(\theta_{A 3}+\theta_{B 2}\right) z}+C_{10} \theta_{A 1} e^{-\theta_{A 1} z}-C_{11} \theta_{A 2} e^{-\theta_{A 2} z}+ \\
& C_{12} \theta_{A 3} e^{-\theta_{A 3} z}+C_{13} \theta_{B 1} e^{-\theta_{B 1} z}-C_{14} \theta_{B 2} e^{-\theta_{B 2} z}+C_{15} \theta_{B 3} e^{-\theta_{B 3} z},
\end{aligned}
$$

where $C_{i}, i=1 \ldots \ldots 9$ are given by $(5)$ and

$$
\begin{aligned}
& C_{10}=\frac{\theta_{A 2} \theta_{B 3}}{\left(\theta_{A 2}-\theta_{A 1}\right)\left(\theta_{A 3}-\theta_{A 1}\right)}, \quad C_{11}=\frac{\theta_{A 1} \theta_{A 3}}{\left(\theta_{A 2}-\theta_{A 1}\right)\left(\theta_{A 3}-\theta_{A 2}\right)}, \\
& C_{12}=\frac{\theta_{A 1} \theta_{A 2}}{\left(\theta_{A 3}-\theta_{A 2}\right)\left(\theta_{A 3}-\theta_{A 1}\right)}, \quad C_{13}=\frac{\theta_{B 2} \theta_{B 3}}{\left(\theta_{B 2}-\theta_{B 1}\right)\left(\theta_{B 3}-\theta_{B 1}\right)}, \\
& C_{14}=\frac{\theta_{B 1} \theta_{B 3}}{\left(\theta_{B 2}-\theta_{B 1}\right)\left(\theta_{B 3}-\theta_{B 2}\right)}, \quad C_{15}=\frac{\theta_{B 1} \theta_{B 2}}{\left(\theta_{B 3}-\theta_{B 2}\right)\left(\theta_{B 3}-\theta_{B 1}\right)} .
\end{aligned}
$$


As proceeding in case (i), we get

$$
\begin{aligned}
E(T) & =C_{10} E_{\theta_{A 1}}-C_{11} E_{\theta_{A 2}}+C_{12} E_{\theta_{A 3}}+C_{13} E_{\theta_{B 1}}-C_{14} E_{\theta_{B 2}} \\
& +C_{15} E_{\theta_{B 3}}-C_{1} E_{\theta_{A 1}, \theta_{B 1}}-C_{2} E_{\theta_{A 2}, \theta_{B 2}}-C_{3} E_{\theta_{A 3}, \theta_{B 3}} \\
& +C_{4} E_{\theta_{A 1}, \theta_{B 2}}-C_{5} E_{\theta_{A 1}, \theta_{B 3}} \\
& +C_{6} E_{\theta_{A 2}, \theta_{B 1}}+C_{7} E_{\theta_{A 2}, \theta_{B 3}}-C_{8} E_{\theta_{A 3}, \theta_{B 1}}+C_{9} E_{\theta_{A 3}, \theta_{B 2}}
\end{aligned}
$$

and

$$
\begin{aligned}
E\left(T^{2}\right) & =C_{10} E_{\theta_{A 1}}^{2}-C_{11} E_{\theta_{A 2}}^{2}+C_{12} E_{\theta_{A 3}}^{2}+C_{13} E_{\theta_{B 1}}^{2}-C_{14} E_{\theta_{B 2}}^{2} \\
& +C_{15} E_{\theta_{B 3}}^{2}-C_{1} E_{\theta_{A 1}, \theta_{B 1}}^{2}-C_{2} E_{\theta_{A 2}, \theta_{B 2}}^{2}-C_{3} E_{\theta_{A 3}, \theta_{B 3}}^{2} \\
& +C_{4} E_{\theta_{A 1}, \theta_{B 2}}^{2}-C_{5} E_{\theta_{A 1}, \theta_{B 3}}^{2}+C_{6} E_{\theta_{A 2}, \theta_{B 1}}^{2} \\
& +C_{7} E_{\theta_{A 2}, \theta_{B 3}}^{2}-C_{8} E_{\theta_{A 3}, \theta_{B 1}}^{2}+C_{9} E_{\theta_{A 3}, \theta_{B 2}}^{2} .
\end{aligned}
$$

(15) and (16) give the variance of the time to recruitment for this case.

Case (iii)

$$
Z=Z_{A 1}+Z_{A 2}+Z_{A 3}+Z_{B 1}+Z_{B 2}+Z_{B 3}
$$

In this case it can be shown that

$$
\begin{aligned}
k(z)= & C_{16} \theta_{A 1} e^{-\theta_{A 1} z}-C_{17} \theta_{A 2} e^{-\theta_{A 2} z}+C_{18} \theta_{A 3} e^{-\theta_{A 3} z}+C_{19} \theta_{B 1} e^{-\theta_{B 1} z} \\
& -C_{20} \theta_{B 2} e^{-\theta_{B 2} z}+C_{21} \theta_{B 3} e^{-\theta_{B 3} z}
\end{aligned}
$$

where

$$
\begin{aligned}
& C_{16}=\frac{\theta_{A 2} \theta_{A 3} \theta_{B 1} \theta_{B 2} \theta_{B 3}}{\left(\theta_{A 2}-\theta_{A 1}\right)\left(\theta_{A 3}-\theta_{A 1}\right)\left(\theta_{B 1}-\theta_{A 1}\right)\left(\theta_{B 2}-\theta_{A 1}\right)\left(\theta_{B 3}-\theta_{A 1}\right)} \\
& C_{17}=\frac{\theta_{A 1} \theta_{A 3} \theta_{B 1} \theta_{B 2} \theta_{B 3}}{\left(\theta_{A 2}-\theta_{A 1}\right)\left(\theta_{A 3}-\theta_{A 2}\right)\left(\theta_{B 1}-\theta_{A 2}\right)\left(\theta_{B 2}-\theta_{A 2}\right)\left(\theta_{B 3}-\theta_{A 2}\right)} \\
& C_{18}=\frac{\theta_{A 1} \theta_{A 2} \theta_{B 1} \theta_{B 2} \theta_{B 3}}{\left(\theta_{A 3}-\theta_{A 2}\right)\left(\theta_{A 3}-\theta_{A 1}\right)\left(\theta_{B 1}-\theta_{A 3}\right)\left(\theta_{B 2}-\theta_{A 3}\right)\left(\theta_{B 3}-\theta_{A 3}\right)} \\
& C_{19}=\frac{\theta_{A 1} \theta_{A 2} \theta_{A 3} \theta_{B 2} \theta_{B 3}}{\left(\theta_{B 1}-\theta_{A 1}\right)\left(\theta_{B 1}-\theta_{A 2}\right)\left(\theta_{B 1}-\theta_{A 3}\right)\left(\theta_{B 2}-\theta_{B 1}\right)\left(\theta_{B 3}-\theta_{B 1}\right)} \\
& C_{20}=\frac{\theta_{A 1} \theta_{A 2} \theta_{A 3} \theta_{B 1} \theta_{B 3}}{\left(\theta_{B 2}-\theta_{A 1}\right)\left(\theta_{B 2}-\theta_{A 2}\right)\left(\theta_{B 2}-\theta_{A 3}\right)\left(\theta_{B 2}-\theta_{B 1}\right)\left(\theta_{B 3}-\theta_{B 2}\right)} \\
& C_{21}=\frac{\theta_{A 1} \theta A 2 \theta_{A 3} \theta_{B 1} \theta_{B 2}}{\left(\theta_{B 3}-\theta_{A 1}\right)\left(\theta_{B 3}-\theta_{A 2}\right)\left(\theta_{B 3}-\theta_{A 3}\right)\left(\theta_{B 3}-\theta_{B 1}\right)\left(\theta_{B 3}-\theta_{B 2}\right)} \\
& E(T)=C_{16} E_{\theta_{A 1}}-C_{17} E_{\theta_{A 2}}+C_{18} E_{\theta_{A 3}}+C_{19} E_{\theta_{B 1}}-C_{20} E_{\theta_{B 2}}+C_{21} E_{\theta_{B 3}}
\end{aligned}
$$


and

$$
\begin{aligned}
E\left(T^{2}\right)=C_{16} E_{\theta_{A 1}}^{2}-C_{17} E_{\theta_{A 2}}^{2}+C_{18} E_{\theta_{A 3}}^{2} & \\
& +C_{19} E_{\theta_{B 1}}^{2}-C_{20} E_{\theta_{B 2}}^{2}+C_{21} E_{\theta_{B 3}}^{2} .
\end{aligned}
$$

In the equations (13), (15) and (17)

$$
\begin{array}{r}
E_{\alpha, \beta}=\frac{C_{1}\left[1-\bar{f}\left(\mu_{2}\left(1-\bar{h}_{A}(\alpha+\beta) \bar{h}_{B}(\alpha+\beta)\right)\right)\right]}{\mu_{2}\left(1-\bar{h}_{A}(\alpha+\beta) \bar{h}_{B}(\alpha+\beta)\right)\left[1-\bar{f}\left(\mu_{2}\left(1-\bar{h}_{A}(\alpha+\beta) \bar{h}_{B}(\alpha+\beta)\right)\right)\right.} \\
\left.\bar{g}_{A}(\alpha+\beta) \bar{g}_{B}(\alpha+\beta)\right]
\end{array}
$$

and

$$
E_{\alpha}=\frac{C_{1}\left[1-\bar{f}\left(\mu_{2}\left(1-\bar{h}_{A}(\alpha) \bar{h}_{B}(\alpha)\right)\right)\right]}{\mu_{2}\left(1-\bar{h}_{A}(\alpha) \bar{h}_{B}(\alpha)\right)\left[1-\bar{f}\left(\mu_{2}\left(1-\bar{h}_{A}(\alpha) \bar{h}_{B}(\alpha)\right)\right) \bar{g}_{A}(\alpha) \bar{g}_{B}(\alpha)\right]} .
$$

In the equation (14), (16) and (18)

$$
\begin{array}{r}
C_{1}\left[2 \mu_{2}\left(1-\bar{h}_{A}(\alpha+\beta) \bar{h}_{B}(\alpha+\beta)\right)\left(1-\bar{g}_{A}(\alpha+\beta) \bar{g}_{B}(\alpha+\beta)\right)\right. \\
E_{\alpha, \beta}^{2}=\frac{\bar{f}_{A}\left(\mu_{2}\left(1-\bar{h}_{A}(\alpha+\beta) \bar{h}_{B}(\alpha+\beta)\right)\right)+2\left[1-\bar{f}\left(\mu_{2}\left(1-\bar{h}_{A}(\alpha+\beta) \bar{h}_{B}(\alpha+\beta)\right)\right)\right.}{\left.\left.\bar{g}_{A}(\alpha+\beta) \bar{g}_{B}(\alpha+\beta)\right]\left[1-\bar{f}\left(\mu_{2}\left(1-\bar{h}_{A}(\alpha+\beta) \bar{h}_{B}(\alpha+\beta)\right)\right)\right]\right]} \\
\mu_{2}^{2}\left(1-\bar{h}_{A}(\alpha+\beta) \bar{h}_{B}(\alpha+\beta)\right)^{2}\left[1-\bar{f}\left(\mu_{2}\left(1-\bar{h}_{A}(\alpha+\beta) \bar{h}_{B}(\alpha+\beta)\right)\right)\right. \\
\left.\bar{g}_{A}(\alpha+\beta) \bar{g}_{B}(\alpha+\beta)\right]^{2}
\end{array}
$$

and

$$
\begin{array}{r}
C_{1}\left[2 \mu_{2}\left(1-\bar{h}_{A}(\alpha) \bar{h}_{B}(\alpha)\right)\left(1-\bar{g}_{A}(\alpha) \bar{g}_{B}(\alpha)\right) \bar{f}^{\prime}\left(\mu_{2}\left(1-\bar{h}_{A}(\alpha) \bar{h}_{B}(\alpha)\right)\right)\right. \\
E_{\alpha}^{2}=\frac{\left.+2\left[1-\bar{f}\left(\mu_{2}\left(1-\bar{h}_{A}(\alpha) \bar{h}_{B}(\alpha)\right)\right) \bar{g}_{A}(\alpha) \bar{g}_{B}(\alpha)\right]\left[1-\bar{f}\left(\mu_{2}\left(1-\bar{h}_{A}(\alpha) \bar{h}_{B}(\alpha)\right)\right)\right]\right]}{\mu_{2}^{2}\left(1-\bar{h}_{A}(\alpha) \bar{h}_{B}(\alpha)\right)^{2}\left[1-\bar{f}\left(\mu_{2}\left(1-\bar{h}_{A}(\alpha) \bar{h}_{B}(\alpha)\right)\right) \bar{g}_{A}(\alpha) \bar{g}_{B}(\alpha)\right]^{2}} .
\end{array}
$$

(17) and (18) give the variance of the time to recruitment for this case.

Special case. Suppose $X_{A i}, X_{B i}, Y_{A j}$ and $Y_{B j}$ follow exponential distribution with parameters $\alpha_{1 A}, \alpha_{1 B}, \alpha_{2 A}$ and $\alpha_{2 B}$, respectively.

In this case

$$
\begin{array}{ll}
\bar{g}_{A}(\theta)=\frac{\alpha_{1 A}}{\alpha_{1 A}+\theta}, & \bar{g}_{B}(\theta)=\frac{\alpha_{1 B}}{\alpha_{1 B}+\theta}, \\
\bar{h}_{A}(\theta)=\frac{\alpha_{2 A}}{\alpha_{2 A}+\theta}, & \bar{h}_{A}(\theta)=\frac{\alpha_{2 B}}{\alpha_{2 B}+\theta} .
\end{array}
$$


Using (19) in (13), (15), (17), (14),(16) and (18), we get the analytical results for the mean and variance of the time to recruitment for all the cases in closed form.

Note. The results for the performance measures corresponding to cases (i), (ii) and (iii) when the inter-policy decisions times are independent and identically distributed exponential random variables can be deduced from our results for the respective cases by taking $p=1$.

\section{Numerical Illustration}

The mean and variance of time to recruitment for all the cases are numerically illustrated by varying one parameter and keeping all the other parameters fixed. The effect of the nodal parameters $\alpha_{1 A}, \alpha_{1 B}, \alpha_{2 A}, \alpha_{2 B}, \mu_{h}, \mu_{l}, \mu_{2}$ and $p$ on the mean and variance of time to recruitment are shown in the following table.

Table $1: \mu_{h}=2 ; \mu_{l}=4 ; \mu_{2}=2 ; p=0.07 ; \theta_{A 1}=0.002 ;$

$\theta_{A 2}=0.01 ; \theta_{A 3}=0.03 ; \theta_{B 1}=0.07 ; \theta_{B 2}=0.04 ; \theta_{B 3}=0.05$

\begin{tabular}{||c|c|c|c|c|c|c|c|c|c||}
\hline \multirow{2}{*}{$\alpha_{1 A}$} & $\alpha_{1 B}$ & $\alpha_{2 A}$ & $\alpha_{2 B}$ & \multicolumn{2}{|c|}{ case(i) } & \multicolumn{2}{c|}{ case(ii) } & \multicolumn{2}{c||}{ case(iii) } \\
\cline { 5 - 10 } & & & & $\mathrm{E}(\mathrm{T})$ & $\mathrm{V}(\mathrm{T})$ & $\mathrm{E}(\mathrm{T})$ & $\mathrm{V}(\mathrm{T})$ & $\mathrm{E}(\mathrm{T})$ & $\mathrm{V}(\mathrm{T})$ \\
\hline \hline 0.1 & 0.2 & 0.1 & 0.5 & 0.844 & 0.453 & 8.063 & 42.84 & 9.181 & 36.80 \\
\hline 0.5 & 0.2 & 0.1 & 0.5 & 1.286 & 0.928 & 12.79 & 107.4 & 14.52 & 93.28 \\
\hline 0.9 & 0.2 & 0.1 & 0.5 & 1.378 & 1.058 & 13.69 & 123.2 & 15.55 & 106.9 \\
\hline 0.1 & 0.1 & 0.2 & 0.5 & 0.785 & 0.417 & 7.420 & 33.66 & 8.329 & 30.09 \\
\hline 0.1 & 0.5 & 0.2 & 0.5 & 1.118 & 0.740 & 11.11 & 74.84 & 12.42 & 67.98 \\
\hline 0.1 & 0.9 & 0.2 & 0.5 & 1.181 & 0.818 & 11.77 & 83.86 & 13.16 & 76.33 \\
\hline 0.2 & 0.1 & 0.1 & 0.5 & 0.844 & 0.453 & 8.063 & 42.84 & 9.181 & 36.80 \\
\hline 0.2 & 0.1 & 0.5 & 0.5 & 1.047 & 0.684 & 10.36 & 61.03 & 11.45 & 57.51 \\
\hline 0.2 & 0.1 & 0.9 & 0.5 & 1.080 & 0.732 & 10.72 & 63.49 & 11.78 & 60.85 \\
\hline 0.2 & 0.1 & 0.5 & 0.1 & 1.022 & 0.641 & 9.474 & 67.58 & 11.10 & 53.84 \\
\hline 0.2 & 0.1 & 0.5 & 0.5 & 1.286 & 0.928 & 12.79 & 107.4 & 14.52 & 93.28 \\
\hline 0.2 & 0.1 & 0.5 & 0.9 & 1.330 & 0.986 & 13.31 & 114.02 & 15.05 & 100.2 \\
\hline
\end{tabular}


Table $2: \alpha_{1 A}=0.5 ; \alpha_{1 B}=0.5 ; \alpha_{2 A}=0.2 ; \alpha_{2 B}=0.1 ; \theta_{A 1}=0.002$;

$\theta_{A 2}=0.01 ; \theta_{A 3}=0.03 ; \theta_{B 1}=0.07 ; \theta_{B 2}=0.04 ; \theta_{B 3}=0.05$

\begin{tabular}{||c|c|c|c|c|c|c|c|c|c||}
\hline \multirow{2}{*}{$\mu_{h}$} & $\mu_{l}$ & $\mu_{2}$ & $p$ & \multicolumn{2}{|c|}{ case(i) } & \multicolumn{2}{c|}{ case(ii) } & \multicolumn{2}{c||}{ case(iii) } \\
\cline { 5 - 10 } & & & & $\mathrm{E}(\mathrm{T})$ & $\mathrm{V}(\mathrm{T})$ & $\mathrm{E}(\mathrm{T})$ & $\mathrm{V}(\mathrm{T})$ & $\mathrm{E}(\mathrm{T})$ & $\mathrm{V}(\mathrm{T})$ \\
\hline \hline 0.1 & 1.5 & 2.0 & 0.07 & 1.924 & 2.302 & 18.30 & 281.7 & 21.85 & 218.7 \\
\hline 0.5 & 1.5 & 2.0 & 0.07 & 1.889 & 2.148 & 17.49 & 244.8 & 20.78 & 189.3 \\
\hline 0.9 & 1.5 & 2.0 & 0.07 & 1.872 & 2.091 & 17.31 & 237.1 & 20.52 & 184.1 \\
\hline 0.5 & 1.1 & 2.0 & 0.07 & 1.970 & 2.350 & 17.88 & 270.5 & 21.54 & 202.7 \\
\hline 0.5 & 1.5 & 2.0 & 0.07 & 1.889 & 2.148 & 17.49 & 244.8 & 20.78 & 189.3 \\
\hline 0.5 & 1.9 & 2.0 & 0.07 & 1.818 & 1.986 & 17.08 & 226.1 & 20.12 & 178.5 \\
\hline 1.5 & 1.2 & 0.1 & 0.7 & 2.998 & 5.202 & 30.25 & 496.3 & 33.14 & 481.8 \\
\hline 1.5 & 1.2 & 0.5 & 0.7 & 2.372 & 3.315 & 23.04 & 341.27 & 26.09 & 297.9 \\
\hline 1.5 & 1.2 & 0.9 & 0.7 & 1.963 & 2.295 & 18.31 & 255.5 & 21.51 & 202.3 \\
\hline 0.7 & 1.5 & 2.0 & 0.01 & 1.861 & 2.063 & 17.23 & 233.8 & 20.41 & 181.9 \\
\hline 0.7 & 1.5 & 2.0 & 0.05 & 1.873 & 2.097 & 17.33 & 238.1 & 20.55 & 184.7 \\
\hline 0.7 & 1.5 & 2.0 & 0.09 & 1.885 & 2.130 & 17.42 & 242.1 & 20.68 & 187.2 \\
\hline
\end{tabular}

From the above tables the following inference are presented which agree with reality:

1. When $\alpha_{1 A}$ increases and keeping all the other parameters fixed, the mean and variance of time to recruitment increase for all the three cases.

2. When $\alpha_{1 B}$ increases and keeping all the other parameters fixed, the mean and variance of time to recruitment increase for all the three cases.

3. When $\alpha_{2 A}$ increases and keeping all the other parameters fixed, the mean and variance of time to recruitment increase for all the three cases.

4. When $\alpha_{2 B}$ increases and keeping all the other parameters fixed, the mean and variance of time to recruitment increase for all the three cases.

5. When $\mu_{h}$ increases and keeping all the other parameters fixed, the mean and variance of time to recruitment decrease for all the three cases.

6. When $\mu_{l}$ increases and keeping all the other parameters fixed, the mean and variance of time to recruitment decrease for all the three cases.

7. When $\mu_{2}$ increases and keeping all the other parameters fixed, the mean and variance of time to recruitment decrease for all the three cases. 
8. When $p$ increases and keeping all the other parameters fixed, the mean and variance of time to recruitment increase for all the three cases.

\section{Conclusion}

The manpower model developed in this paper accommodates an important nodal parameter namely attrition rate with respective proportion and this aspect makes the manpower problem more realistic compared to the stochastic models in the literature confined to the present study. The findings reported in this paper will be very useful in planning recruitments in future for those marketing organizations with depletion of manpower due to attrition. The goodness of fit for the distributions assumed in this paper can be examined by collecting relevant data and using simulation the applicability of the developed model can be studied.

\section{References}

[1] G.Arivazhagan, R.Elangovan and R.Sathiyamoorthi, Determination of expected time to recruitment when the breakdown threshold has three components, Int.J.Agricult.Stat.Sci, 6 No.1(2010),203-213.

[2] S.Dhivya, and A.Srinivasan, Stochastic model for time to recruitment Under two sources of depletion Of manpower using univariate Policy of recruitment,International J. of Multidispl. Research 83 Advcs. in Engg, 5 No.4(2013),17-26.

[3] S.Dhivya and A.Srinivasan, Stochastic model for time to recruitment under two Sources of depletion of manpower associated with different renewal process, International Journal of Revolution in Science and Humanity, $\mathbf{2}$ No.1(2013),45-51.

[4] S.Dhivya and A.Srinivasan, Estimation of Variance of the time to recruitment under two sources of Depletion, International Journal of Advanced Computer Research,3 No.4(2013),74-78.

[5] S.Dhivya and A.Srinivasan, Variance of the time to recruitment for a two grade Manpower system with two sources of depletion, Asian Academic Research Journal of Social Science \&3 Humanities, 1(2014),258-266. 
[6] R.Elangovan , R.Sathiyamoorthi and E.Susiganeshkumar, Estimation of expected time to recruitment under two sources of depletion of manpower, Proceedings of the International Conference on Stochastic Modelling and Simulation, Allied Publishers Pvt.Ltd.,Chennai,(2011),74-78.

[7] Karlin, Samuel and Taylor, M.Haward., A First Course in Stochastic Processes, New York, NY:Academic Press,Second Edition,(1975).

[8] C.Sathiya and A.Srinivasan, A stochastic model for the time to Recruitment in a single grade manpower system under two sources of deplietions of manpower using univariate recruitment policy, Cayley J.Math., 3 No.1(2014),13-20.

[9] S.Suganya and A.Srinivasan, A stochastic model for the time to Recruitment in a two grade manpower system under two sources of deplietion of manpower using univariate recruitment policy, Bessel J.Math., 4 No.2(2014),57-67. 